\title{
Autosomal dominant dopa-responsive dystonia
}

INSERM

\section{Source}

INSERM. (1999). Orphanet: an online rare disease and orphan drug data base. Autosomal dominant dopa-responsive dystonia. ORPHA:98808

Autosomal dominant dopa-responsive dystonia (DYT 5a) is a rare neurometabolic disorder characterized by childhood-onset dystonia that shows a dramatic and sustained response to low doses of levodopa (L-dopa) and that may be associated with parkinsonism at an older age. 